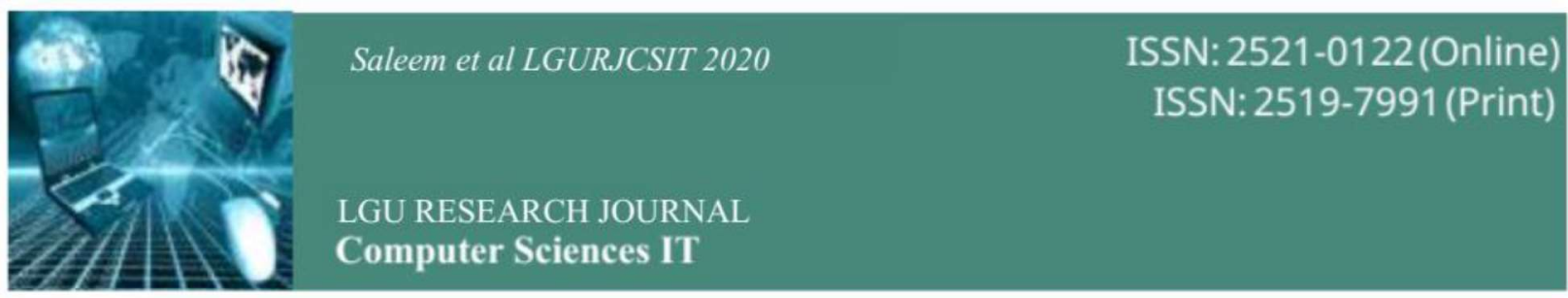

Vol.4 Issue3, July-Sep 2020

\title{
Flow-Based Rules Generation for Intrusion Detection System using Machine Learning Approach
}

\author{
Yasir Saleem ${ }^{1}$, Usama Anwar ${ }^{1}$, Muhammad Khawar Bashir ${ }^{2}$, Sheraz Naseer ${ }^{3}$ Nadia Tabassum ${ }^{4}$ \\ ${ }^{1}$ Department of Computer Science and Engineering, University of Engineering, Lahore. \\ 2 Department of Statistics and Computer Science, University of Veterinary and Animal Sciences. \\ ${ }^{3}$ Department of Computer Science, University of Management and Technology. \\ ${ }^{4}$ Virtual university of Pakistan
}

\begin{abstract}
Rapid increase in internet users also brought new ways of privacy and security exploitation. Intrusion is one of such attacks in which an authorized user can access system resources and is major concern for cyber security community. Although AV and firewall companies work hard to cope with this kind of attacks and generate signatures for such exploits but still, they are lagging behind badly in this race. This research proposes an approach to ease the task of rules generation by making use of machine learning for this purpose. We used 17 network features to train a random forest classifier and this trained classifier is then translated into rules which can easily be integrated with most commonly used firewalls like snort and suricata etc. This work targets five kind of attacks: brute force, denial of service, HTTP DoS, infiltrate from inside and SSH brute force. Separate rules are generated for each kind of attack. As not every generated rule contributes towards detection that's why an evaluation mechanism is also used which selects the best rule on the basis of precision and f-measure values. Generated rules for some attacks have $100 \%$ precision with detection rate of more than $99 \%$ which represents effectiveness of this approach on traditional firewalls. As our proposed system translates trained classifier model into set of rules for firewalls so it is not only effective for rules generation but also give machine learning characteristics to traditional firewall to some extent.
\end{abstract}

Keywords: Intrusion detection system, automatic rules generation, flow-based rules, computer security, data mining in cyber security. 


\section{INTRODUCTION}

Number of devices connected to internet are increasing rapidly. Majority of people are using electronic devices for their daily activities but with this wide spread of internet, number of malicious and unwanted activities is also being increased. These activities put privacy and security of end-user's data at risk. Data leakage can happen when an un-authorized user has access to the system or an authorized user have access to un-authorized portions or files of system. This kind of access to system is called intrusion and Intrusion Detection System (IDS) is used to detect such operations and protect users from data loss.

In cyber security community, intrusions are of majors concerns these days. Although anti-virus and firewall companies are trying hard to protect users against such attacks by generating efficient signatures for new attacks but they are lagging badly in this race as signature generation is very time-consuming task and comparing payload of each network packet for finding these signatures also requires very high computation power.

Network attacks are evolving with time and according to reports of AV-Test institute from 2016, 390000 malware samples are registered on daily basis [3]. It is very time-consuming and tedious to analyze each sample and generate signature for it manually. This failure of traditional approaches called for a mechanism which can cope with the problem of rules generation by monitoring and generating rules automatically. Machine learning approach can be used in this regard efficiently and help in coping with this problem. This research focuses on a system which can ease the task of rules generation for firewalls by making use of machine learning algorithms. The proposed approach doesn't only provide mechanism for automation of rules generation but also proposes an approach of flow-based detection which is much efficient and requires low computation power as compared to content based detection system.

The purpose of this research is to efficiently generate intrusion detection rules for firewall by using machine learning and a fairly large and uniform dataset which is very close to real-life scenarios. Instead of producing content-based rules, flow-based rules are generated to reduce computation requirements for rule matching and detection. A filtering mechanism is also introduced to select the rules which covers maximum scenarios with best precision to reduce number of rules for firewall.

In section 2., related work is reviewed. Section 3. presents our proposed architecture and its implementation. Section 4. presents evaluation criteria and our obtained results and in section 5., conclusion and future work is discussed.

\section{RELATED WORK}

In today's connected world, end-users are at highest risk of getting their systems compromised. It can be done by iframe-injection, phishing [25], a drive-by download [32] or malware. Intrusion is also form of security breach in which an un-authorized user can get access to one's system or an authorized user can perform un-authorized operation on others' system. Intrusion detection systems are the systems which not only detect such security violations but also alerts the user about such activities.

An intrusion Detection System (IDS) is a software or system which monitors network traffic for anomalous behavior or policy violation and alerts the user or admin about such activities. First intrusion detection system was developed by Anderson in 1980 [2] which was further improved by Denning in 1987 [13]. It was an IDS framework which detected security violations by monitoring audit records of system for specific patterns. With passage of time, intrusion attack has become more and more advance and have capability to modify system records etc. to remain un-detected. Anti-viruses and firewalls are used for prevention of servers and systems but these kinds of sophisticated attacks can happen in fraction of seconds and require more sophisticated approaches to counter them.

On the base of ways how IDSs work, they are categorized in to two types: Host based Intrusion Detection System (HIDS) and Network based Intrusion Detection System (NIDS) [39, 61-64]. HIDS are normally installed on end-user machines and protect the user from un-authorized access or prevents user's information from destruction. This kind of access may include privilege escalation, manipulation of system logs, system calls modification or access to sensitive files. HIDS provides protection against such attacks by monitoring every activity on host system [7]. On contrary to HIDS, NIDS are deployed on the network and gathers network information for analysis. They are normally 
installed a multiple point on network and gathered information is sent to central database which further use different detection methods on provided data.

\section{A. Research Related to Detection Approaches}

On base of detection method, IDS are further divided in to two categories: Anomaly based detection $[20,4]$ and signature-based detection $[58,60]$. An anomaly represents a deviation from normal behavior. By monitoring network activity over a period of time, a normal behavior can be defined on the basis of bandwidth consumed, protocols or ports used etc. This is termed as expected behavior of network and anything deviating from it is termed as anomalous and reported to admin. A number of different profiles are created based on different users' behavior and each profile can be static or dynamic [33]. Anomaly detection is also called behavior detection. If profiles are not properly, system may alert admin about legitimate traffic. Such alerts are called false positives. Matthew and Philip [37] showed that such false positives are main problem on anomaly-based IDS. According to Dina et al. [24] and Roberto et al. [15] anomalybased systems have advantage over signaturebased system that they have capability to detect zero-day attack. An anomaly detection system must be trained carefully with valid data before use due to which it' deployment is very complex and difficult.

A number of parameters can be used to define profiles for anomaly detection and using flow parameters is one of them. Some special systems provide flow information like start time, end time, duration, protocol etc. for each IP address pair and port number in the form of IPFIX [47] or Netflow [10,11] records for further analysis of data. Xing et al. suggested OpenFlow and Snort based system called 'Snort Flow' which enables intrusion detection for cloud-based system [59]. In case of attack, it reconfigures the cloud network on-the-fly and deploys countermeasures to prevent attack. Sperotto et al. suggested that flow-based detection system isn't replacement of content-based detection but should be complementary with it [54] thus provide two stage detection. First stage detects some attacks using flow-based detection and then contentbased detection provides further protection.

On contrary to anomaly-based detection, signature-based detection method matches patterns with pattern of known attacks [33]. As known knowledge about an attack is used for pattern matching due to which it is also called knowledge-based detection or misuse detection. It is the most effective and simplest method for detecting known attacks. Misuse or signaturebased detection method manages a database of patterns or signatures of known attacks and analyses whole packet for signature matching. It raises the alarm only if a pattern matching to a known attack is found [4]. In this way, this method solves the problem of false alarms which is present in anomaly-based detection system. Hubballi et al. [26] discussed a number of different techniques which are used to further minimize false alarms in misuse detection. To keep the database of attack signatures updated with new exploits is really challenging due to which it suffers some lag and is not good enough to detect zero-day attacks due to which it suffers from problem of false negatives [30]. But the use of machine learning for generating signatures for new attacks can solve this lag problem [45].

Content-based detection is type signature-based detection in which content of network packets is monitored for pattern matching of known attacks. It works at network platform and scan/monitor every packet and analyze it payload for every network layer for detection of any intrusion or malware [48]. Traditional IDSs like snort [46], suricata [40] and bro [42] inspect payload of each packets for detection purpose. This is a very good approach and can detect any known attack but packet inspection takes too much time and requires very efficient string matching algorithms. Fisk and Varghese [19] suggested a fast string matching algorithm but still inspecting each packets on high speed network becomes impossible without high speed computing [31, 22].

\section{B. Research Related to Machine Learning in Cyber Security}

Machine learning technique is widely used in cybersecurity specially in IDS. In 2001, Australian department of defense carried out a study to explore the possibilities of deployment of ML in IDS. According to this study, ML techniques are very effective in intrusion detection [1]. Although it cannot provide realtime results because it requires sufficient data and analyses to produce results but it can be termed as near real-time operation. A number of machine learning classifiers are used in IDSs like decision 
tree, State Vector Machine (SVM) and neural networks but decision trees are found more effective [16, 28]. Performance of classifier highly depends on data provided for training so data set must be uniform and have real-time traffic. In order to train classifier for IDS, NSLKDD data set [55] is widely used. This dataset has 41 features but not all features are useful and features which are contributing towards prediction are selected. L. Dhanabal and S.P. Shantharajah achieved accuracy of

$99.8 \%, 98.8 \%$ and $74.9 \%$ with J48, SVM and Nave Bayes classifiers respectively by using just 6 features [14]. A.K. Shrivas and A.K. Dewangan used Bayesian Net with Gain Ratio (GR) feature selection technique along with ensemble of Artificial Neural Network (ANN) on KDD99 and NSL-KDD data sets [52]. GR feature selection is used to select relevant features and ANN ensemble is used for detection purposes and achieved accuracy of $98.07 \%$ and $97.78 \%$ with 35 and 29 features respectively. S. Paliwal and R.Gupta also used NSL-KDD data set with genetic algorithm for detection of Remote to User (R2L), probing and DoS attacks with accuracy of more than 97\% [41]. D. P. Gaikwad and R. C. Thool presented in their work that bagging ensemble method for ML classification is much effective than others in terms of efficiency [21].

In addition to supervised learning models, unsupervised learning models are also developed which uses unlabeled data for classification purposes. Jabbar et al. proposed ensemble of ADTree and K-mean clustering for detection purpose and achieved accuracy of $99.8 \%$ [27]. Lin et al. also proposed un-supervised learning method using cluster center and nearest neighbor (CANN) approach [34]. This approach measures two distances: one from cluster center to data sample and other from data to nearest neighbor. Some of these two distances is used by k-nearest neighbor (KNN) for detection purpose. Chae et al. suggested that some redundant and irrelevant feature can result lengthy detection process with performance degradation [8]. In order to reduce data, they proposed new feature selection methods which uses average of total and each class data. Farissi et al. analyzed KDD parameters for neural network classifiers and found that some parameters are meaningless and increase detection rate [17]. This study consists on selecting more efficient parameters based on their performance and utility to optimize IDS performance. Y.P. Raiwani and S.S. Panwar used
Correlation-based Feature Selection (CFS) along with ANN and showed that feature reduction can increase storage efficiency and reduce cost [44]. H. H. Chou and S. D. Wang proposed an adaptive IDS for cloud environment which adapts the ever-changing network and uses decision tree for this purpose [9]. The proposed approach constructs decision tree from un-labelled data using unsupervised learning algorithm called spectral clustering. This solution can easily be deployed in cloud. BRAIN is also an adaptive IDS which uses hardware performance counter and models application behavior using low level hardware events [29]. It uses SVM to detect DoS and DDoS attack by combining modelled application behavior and network statistics.

\section{Research Related to Automated Rules Generation}

It is very time-consuming and tedious task to analyze each malicious sample and generate signature for it manually. Also generating a separate signature for each sample will create a very large signature database which will require much computing power for pattern matching. To solve this problem, automatics rules generation systems are created which analyze malware samples and create efficient and generic rule automatically.

Shim et al. [50] suggested sequence pattern algorithm to generate common substring and extract the header information and location of signature to create snort rules. Nebula is another rule generation system which uses autonomous attack classification [57]. Malware samples are classified in to different classes and rules for each class are generated with positional information of signature to improve performance. Godefroy et al. suggested an approach to automatically create co-related rules [23]. A human expert provides action tree derived from attack tree and a fully automatic system creates co-related rules.

Catania and Garino used machine learning approach to reduce the human effort to create and adjust rules while keeping accuracy within certain limits [6]. Fallahi et al. proposed an approach to automate rule generation using logs of performed attacks [18]. They used two ML classifiers Ripper and C5.0 for automatic rules generation and achieved detection rate of more than $99 \%$ for some attacks. 


\section{PROPOSED ARCHITECTURE AND IMPLEMENTATION}

Traditional signature-based IDS monitor payload of each network packet for detection of any malicious activity which requires high computational power but same results can be achieved with lower computation power by just analyzing network flows. This research focuses on architecture of such systems and proposes a flow-based detection approach. The goal of proposed system is to monitor the network traffic flows and make use of machine learning approach to detect whether a flow is related to intrusion or not. On the base of this detection, rules are generated for signature-based systems like snort [46] or suricata [40] etc. This system is not an alternative to traditional signature-based systems but aids such systems by automatically generating rules for intrusion related flows.

To realize an efficient rules generation system, network traffic must be captured, distinct flows are identified and features required for intrusion detection are extracted to create dataset. On basis of extracted features, some new features are also generated to enhance dataset and improve results. A machine learning classifier is used on this dataset for data classification. After classifier training, trained model translated into set of rules for firewall or traditional IDS. The rules generated by the system are filtered using different evaluation criteria like precision and Fmeasure and only the best rules which contribute toward detection of maximum number of presented scenarios are provided to the firewall.

To build an efficient and accurate system using machine learning, it is important to use a dataset which don't only have information about large number of flows but also consist of uniform data comprised of real-life network traffic. A nonuniform dataset can lead to a biased trained model. In [53, 56], it is argued that most important challenge for evaluation of anomalybased detection in the lack of appropriate datasets.

DARPA [35] and NSL-KDD [55] datasets are widely used in intrusion detection domain. In [38, 5], it is discussed that DARPA dataset is some sort of closed lab dataset which doesn't reflect real world conditions. These datasets are older and network attacks are evolving at rapid pace which makes these datasets outdated and ineffective in recent days. So, a relatively newer and dynamically generated dataset is required should reflect trending intrusions and network patterns as they evolve. To avoid such short comings, instead of using these common and widely used datasets, we used ISCX Flow dataset [51] which is a relatively newer dataset and reflects evolving intrusion patterns.

\section{A. Details About ISCX Dataset}

ISCX dataset was produced by University of New Brunswick in 2015 and it not only have detailed description of intrusions but also abstract distribution models for protocols, applications and low-level network entities. It has real traffic for HTTP, SSH, SMTP, FTP, POP3 and IMAP. To supply anomalous portion of dataset, various multi-stage attack scenarios were carried out. We chose this dataset due to following characteristics:

- Realistic network and traffic: A dataset should have realistic traffic without exhibiting any unintended properties both network wise and traffic wise in order to give the clear picture of attack.

- Labeled dataset: For supervised classification and evaluation of detection mechanism, a dataset must be labeled. Both normal and anomalous data is labeled in this dataset.

- Total interaction capture: Maximum amount of information is required by evaluation mechanism to detect anomalous behavior. So, a dataset should have all the network related interactions. As we are dealing with flow-based data so every packet related to a flow is of much importance.

- Diverse intrusion scenarios: In recent years, network attacks have increased in variety, size, frequency and complexity. So, in depth analysis of IP services and applications is needed to detect such attacks. Many labeled datasets failed in this regard and are in effective for evaluating research results but ISCX dataset does very well in this regard. 


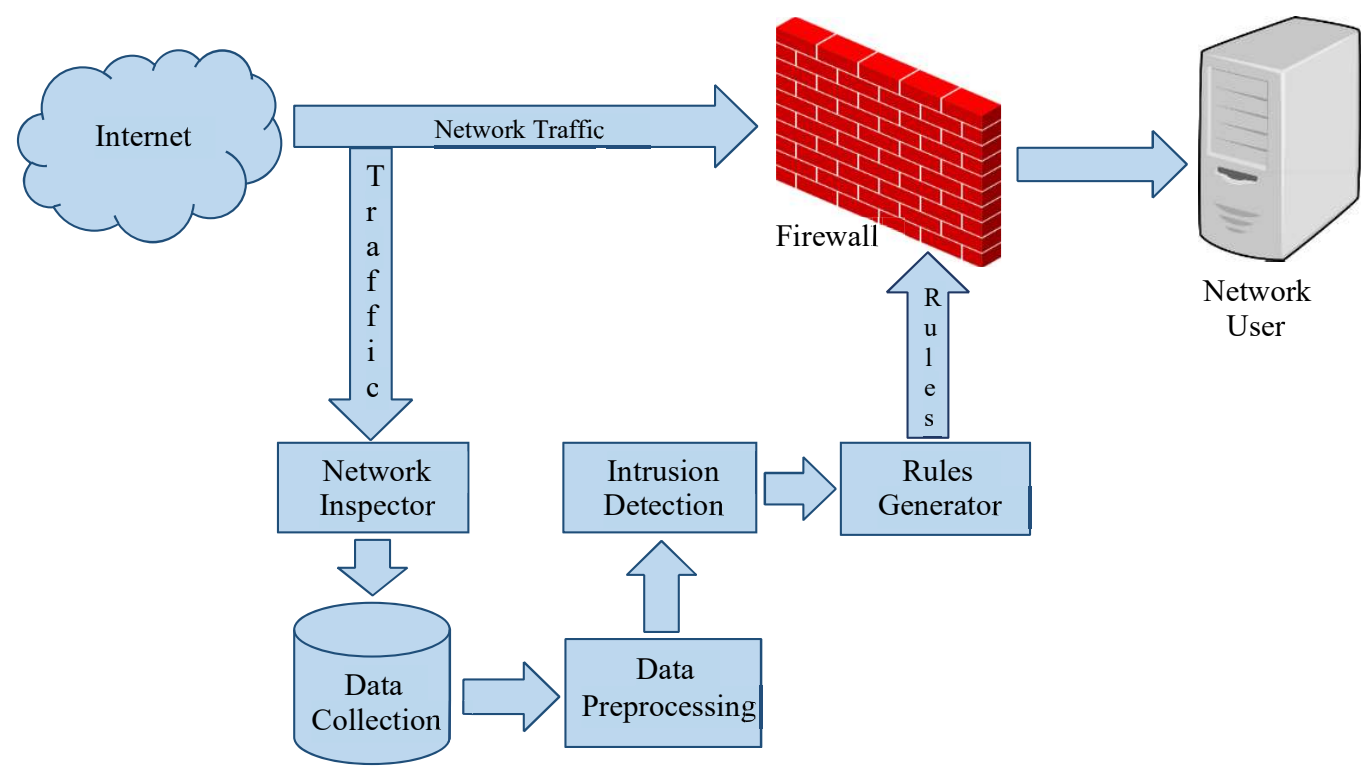

Figure 1: The overall design of proposed IDS

Table 1. Details of flows in ISCX dataset

\begin{tabular}{|c|c|c|c|c|}
\hline Date & Attack Type & $\begin{array}{l}\text { Normal } \\
\text { Flows }\end{array}$ & $\begin{array}{l}\text { Anomalous } \\
\text { Flows }\end{array}$ & Size (GB) \\
\hline $\begin{array}{l}\text { June } \\
12\end{array}$ & Brute Force & 131107 & 2086 & 4.22 \\
\hline $\begin{array}{l}\text { June } \\
13\end{array}$ & Infiltrating the network from inside & 255170 & 20358 & 3.95 \\
\hline $\begin{array}{l}\text { June } \\
14\end{array}$ & HTTP Denial of Service & 167604 & 3776 & 6.85 \\
\hline $\begin{array}{l}\text { June } \\
15\end{array}$ & $\begin{array}{l}\text { Distributed Denial of Service using an IRC } \\
\text { Botnet }\end{array}$ & 391540 & 37460 & 23.4 \\
\hline $\begin{array}{l}\text { June } \\
16\end{array}$ & Brute Force & 522252 & 11 & 17.6 \\
\hline $\begin{array}{l}\text { June } \\
17\end{array}$ & Brute Force SSH & 392376 & 5119 & 12.3 \\
\hline
\end{tabular}

ISCX dataset consists of data for a whole week with each day having separate trend of network traffic. This dataset is comprised of 84 GBs which contains about 2,450,324 flows, out of which 68,810 flows are anomalous. Dataset is captured for $11^{\text {th }}$ June to $17^{\text {th }}$ June and all the network data for 11th June is normal so it isn't considered useful for intrusion detection scenarios. Table 1 gives complete details about each day activity in dataset. Total following five broad categories of attacks are present in this dataset:

\section{B. Feature Extraction}

All data provided by ISCX dataset is in form of raw network capture and we need to extract flow information from it. According to Internet Protocol Flow Information Export (IPFIX), a flow is a set of IP packets passing through an observer point during an interval. All packets in flow set have some common key features. In 
IPFIX terms, these features are called keys of flow. These keys are protocol, source IP, source port, destination ip and destination port [12]. A flow can be unidirectional or bi-directional but we treat all flows as bi-directional because we need features for all packets going from source or coming toward source separately as both have their own significance. The packets with same flow key are bundled to make a complete flow. A flow is considered completed only when either TCP FIN flag appears in packet or time-out happens. In case of TCP, connection is normally terminated by FIN packet and in case of UDP, connection termination happens after timeout. Direction of each packet is also identified on the basis of first packet. Source of first packet is considered as source of flow. After each packet is identified as part of unique flow, important features which are required by classifier are extracted and saved. Table 3 represents list of all the features which are extracted from a flow.

In addition to features extracted from flows, we generated some new features to improve classifier training and to make generated rules more effective and expressive. These features are generated using already extracted features. Table 2 represents all such features. All the features which are used for classification and their descriptions are presented in table 3.

Table 2. Calculation formula for generation of new features

\begin{tabular}{|c|c|}
\hline Generated Features & \begin{tabular}{c} 
Calculation Formula \\
\hline flowDuration
\end{tabular} \\
\hline totalBytes & $\begin{array}{r}\text { totalSourceBytes + } \\
\text { totalDestinationBytes }\end{array}$ \\
\hline totalPackets & $\begin{array}{c}\text { totalSourcePackets + } \\
\text { totalDestinationPackets }\end{array}$ \\
\hline flowBytes/Sec & totalBytes / flowDuration \\
\hline flowPackets/Sec & totalPackets / flowDuration \\
\hline averageBytes/Packet & totalBytes / totalPackets \\
\hline IOByteRate & $\begin{array}{c}\text { totalSourceBytes / } \\
\text { totalDestinationBytes }\end{array}$ \\
\hline IOPacketRate & $\begin{array}{l}\text { totalSourcePackets / } \\
\text { totalDestinationPackets }\end{array}$ \\
\hline
\end{tabular}

\section{Selection of Machine Learning Classifier}

A number of machine learning classifiers are available, each is suitable for different kind of situations. To choose the best suitable classifier, we used five different classifiers from scikit learn [43]. Classifiers used for comparison purposes are logistic regression, Decision Forest (CART), Naive Bayes, Random Forest with 64 CART estimators and Ada Boost. Classifiers are trained using both test train split method and K-fold cross validation method. Both these methods are used for model evaluation of classification problems and provide an indication about the performance of the classifier on unseen data.
In test train split method, dataset is split in to two portions. One is used as train data set and other is used to evaluate the classifier. In $\mathrm{K}$-fold cross validation, data set is divided in to $\mathrm{k}$ equal subsets. $\mathrm{k}-1$ subsets are used to train model and 1 subset is used for evaluation purpose. This procedure is repeated $\mathrm{k}$ times and each sub sample is utilized only once for validation. The results from each of the $\mathrm{k}$ iterations are than averaged to give a single estimate of performance. We used 10-fold cross validation to choose the best classifier for our data. 
Table 3. Flow features and their description

\begin{tabular}{|l|l|}
\hline Feature Name & Description \\
\hline totalSourceBytes & Total bytes flowed from source to destination \\
\hline totalDestinationBytes & Total bytes flowed from destination to source \\
\hline totalDestinationPackets & Total packets flowed from destination to source \\
\hline totalSourcePackets & Total packets flowed from source to destination \\
\hline Direction & Direction of packets \\
\hline sourceTCPFlags & TCP flags used in source packets during whole flow \\
\hline destinationTCPFlags & TCP flags used in destination packets during whole flow \\
\hline protocolName & Protocol name \\
\hline destinationPort & Destination port \\
\hline flowDuration & Duration of flow \\
\hline totalBytes & Total bytes transferred in a flow \\
\hline totalPackets & Total packets transferred in a flow \\
\hline flowBytes/Sec & Average bytes transferred per second \\
\hline flowPackets/Sec & Average packets flow per second \\
\hline averageBytes/Packet & Average bytes in each packet \\
\hline IOByteRate & Ratio of input-output bytes \\
\hline IOPacketRate & Ratio of input-output packets \\
\hline
\end{tabular}

Table 4. Results of various machine learning classifiers

\begin{tabular}{|c|c|c|c|c|}
\hline Classifier & Evaluation Method & Accuracy & Precision & Recall \\
\hline \multirow{2}{*}{$\begin{array}{l}\text { Logistic } \\
\text { Regression }\end{array}$} & Train-test split & 0.93 & 0.51 & 0.38 \\
\hline & $\begin{array}{l}10 \text {-fold cross } \\
\text { validation }\end{array}$ & 0.93 & 0.42 & 0.38 \\
\hline \multirow[b]{2}{*}{ CART } & Train-test split & 0.99 & 0.98 & 0.99 \\
\hline & $\begin{array}{l}10 \text {-fold cross } \\
\text { validation }\end{array}$ & 0.99 & 0.93 & 0.91 \\
\hline \multirow[b]{2}{*}{ Naive Bayes } & Train-test split & 0.80 & 0.23 & 0.81 \\
\hline & $\begin{array}{l}10 \text {-fold cross } \\
\text { validation }\end{array}$ & 0.80 & 0.25 & 0.70 \\
\hline \multirow[b]{2}{*}{ Random Forest } & Train-test split & 0.99 & 0.99 & 0.99 \\
\hline & $\begin{array}{l}10 \text {-fold cross } \\
\text { validation }\end{array}$ & 0.99 & 0.94 & 0.92 \\
\hline \multirow[b]{2}{*}{ Ada Boost } & Train-test split & 0.99 & 0.96 & 0.96 \\
\hline & $\begin{array}{l}10 \text {-fold cross } \\
\text { validation }\end{array}$ & 0.99 & 0.94 & 0.92 \\
\hline
\end{tabular}

Table 4 shows comprehensive comparison of performance of all classifiers. Apart from Naive Bayes classifier, accuracy of all other methods is more than 0.9. Although logistic regression performs better in terms of accuracy, but it's precision and recall parameter are much worse as compared to others. CART, random forest and Ada Boost all performed very well for both traintest split method and 10 -fold cross validation. On basis of these results, we chose random forest 
classifier for rules generation purposes due to following reasons:

1. Random forest gives best performance for our dataset

2. Generation of rules from trained model is relatively easier from a decision tree

3. Random forest generates multiple trees which increases chances of finding more versatile rules

\section{Rules Generation}

Dataset is provided to decision forest classifier for training purposes. After training classifier, a number of decision trees are created. These trees have a number of decision nodes and some leaf nodes. Each decision node represents some criteria on which next decision node is selected. These criteria consist of a feature and a threshold value. If decision criteria are met, right node is selected for further decisions otherwise left node is selected. Leaf nodes have information about final verdict which is normally presented in the form of probability.

To obtained trained model for decision forest classifier, we used python sci-kit learn library [43] which uses CART as base tree classifier. The trained model has many trees and each tree is processed separately. There are two kind of nodes in decision trees. One is decision nodes which have information about features which should be used for decision and a threshold value which decides next node. Others are leaf nodes which gives information about final verdict. It also has information about number of samples which terminated at this node. By using those number of samples, TP and FP can be calculated. As described in [36], decision tree is constructed as:

1. Start from root node

2. For each $X$, find a subset $S$ such that sum of node impurities is minimum in two child nodes. Split $\left\{\mathrm{X}^{*} \in \mathrm{S}^{*}\right\}$ which gives minimum for $\mathrm{S}$ and $\mathrm{X}$ is chosen

3. Apply step 2 to each child node until stopping criteria is reached

A decision tree in CART is generated by first partitioning data in such a way that same labels are grouped together and then subsets are created using split candidates [49].

For training vector $x_{i} \in R^{n}, i=1, \ldots, n$ and label vector $y \in R^{l}$, all space is sampled recursively in such a way that samples with same labels are grouped together. For data in node n, candidate split $\theta=\left(i, t_{m}\right)$ consisting of feature $i$ and threshold $t_{m}$ partitions data in to left and right subset. Let data at node $\mathrm{m}$ is $Q$, then $Q_{\text {left }}(\theta)$ and $Q_{\text {right }}(\theta)$ subsets are calculated as:

$Q_{\text {left }}(\theta)=(x, y) \mid x_{j}<=t_{m}$

$Q_{\text {right }}(\theta)=\frac{Q}{Q_{\text {left }}(\theta)}$

Impurity function $\mathrm{H}()$ is used to fin impurity $\mathrm{m}$. The choice of this function depends on task being carried out either it is classification or regression.

$G(Q, \theta)=\frac{N_{\text {left }}}{N_{m}} H\left(Q_{\text {left }}(\theta)\right)+\frac{N_{\text {right }}}{N_{m}} H\left(Q_{\text {right }}(\theta)\right)$

Parameters which minimize impurity are selected

$\left.\theta^{*}=\operatorname{argmin}_{\theta} G(Q, \theta)\right)$

Recurse on subsets $Q_{\text {lefit }}\left(\theta^{*}\right)$ and $Q_{\text {right }}\left(\theta^{*}\right)$ until stopping criteria is reached. In equation $3, N_{m}<$ $\min _{\text {samples }}$ or $N_{m}=1$

If classification outcome can take values $0,1, \ldots, K$ - 1 then for node $m$ representing region $R_{m}$ with sample observation $N_{m}$, proportion of class $k$ in node $m$ can be represented as

$p_{m k}=\frac{1}{N_{m}} \sum_{x_{i} \in R_{m}} I\left(y_{i}=k\right)$

Now impurity function can be defined as:

$H\left(X_{m}\right)=\sum_{k} p_{m k}\left(1-p_{m k}\right)$

If classification outcome is a continuous value then for node $m$ representing region $R_{m}$ with sample observation $N_{m}$, common goal is to minimize Mean Squared Error,

$c_{m}=\frac{1}{N_{m}} \sum_{i \in N_{m}} y_{i}$

$H\left(X_{m}\right)=\frac{1}{N_{m}} \sum_{i \in N_{m}}\left(y_{i}-c_{m}\right)^{2}$

Starting from root, first of all feature value is extracted from the node. A feature can be continuous or a categorical feature. If the feature is continuous, then for left node rule is created with condition feature value $<=$ threshold value 
and for right node rule is created with condition feature value $>$ threshold value. If feature is categorical, then rule for left node have condition feature value $==$ threshold value and rule for right node have condition feature value $!=$ threshold value. A sample rule is like given below:

"If flowBytesPerSec $<=83657.5$ and destinationPort $>24$ and destinationPort $>443$ and bytesPerPacket $<=64.5$ and sourcePort $>$ 53107 then InfilterateFromInside"

To generate a rule, a tree is iterated from root node to leaf node using DFS method. Once the leaf node is reached, final verdict is extracted from it. Leaf node have information about how many numbers of samples from each kind of verdict fall on this node. This information is encoded in the form of an array like [100 0100 $10]$. This array presents that leaf node had 100 samples for InfiltrateFromInside, 0 sample for Bruteforce, 10 samples for Normal flow, 0 sample for DDos, 1 sample for SSH Bruteforce and 0 sample for HTTP DoS. As it have maximum number of samples for InfiltrateFromInside, so this rules presents this attack where TP is 100 and $\mathrm{FP}$ is 11 . This TP and FP value is further used for evaluation of rules.

\section{EXPERIMENTAL RESULTS AND EVALUATIONS}

To get trained model for rules generation, random forest with 64 trees is generated with each tree have maximum 512 nodes and 16 is used as split criteria for each node. Figure 2 provides information about depth of leaf nodes along with their frequency for each tree. It is clearly visible that mostly leaf nodes are at depth of 11 to 14 for most of the trees but many leaf nodes also have depth up to 16. It means in most of cases, 11 to 14 features are used to reach conclusion.

Converting a trained decision tree into a set of rules results in a very large number of rules. All of these rules are not useful and providing such a large number of rules to firewall effects the performance of firewall and may cause many false positives. Figure 3 provides information about number of samples present in each node. In other words, it gives information about coverage of each leaf node. Most of the leaf nodes on average cover only few hundred samples and rules created on the base of such nodes will be of no use. Only few leaf nodes have coverage for thousands of samples and such kind of leaf nodes are of our interest because these will give the best kind of rules. To filter most effective rules, we proposed an evaluation criteria. So before discussing our achieved results, we will first discuss evaluation criteria.

\section{A. Evaluation Criteria for Generated Rules}

- A set of four equations is used to measure the worth of a rule. Following terminologies are used in these equations:

- True Positive (TP): Samples which are correctly classified as positive. It represents number of flows of specific attack tag, which are correctly classified by generated rules as same type of attack.

- False Positive (FP): Samples which are incorrectly classified as positive. It represents number of normal or malicious flows which are classified incorrectly by generated rule as specified attack.

- True Negative (TN): Samples which are correctly classified as negative. It represents number of normal flows which are correctly classified as normal by generated rule.

- False Negative (FN): Samples which are incorrectly classified as negative. It represents number of malicious flows which are classified incorrectly by generated rule as normal flows. 


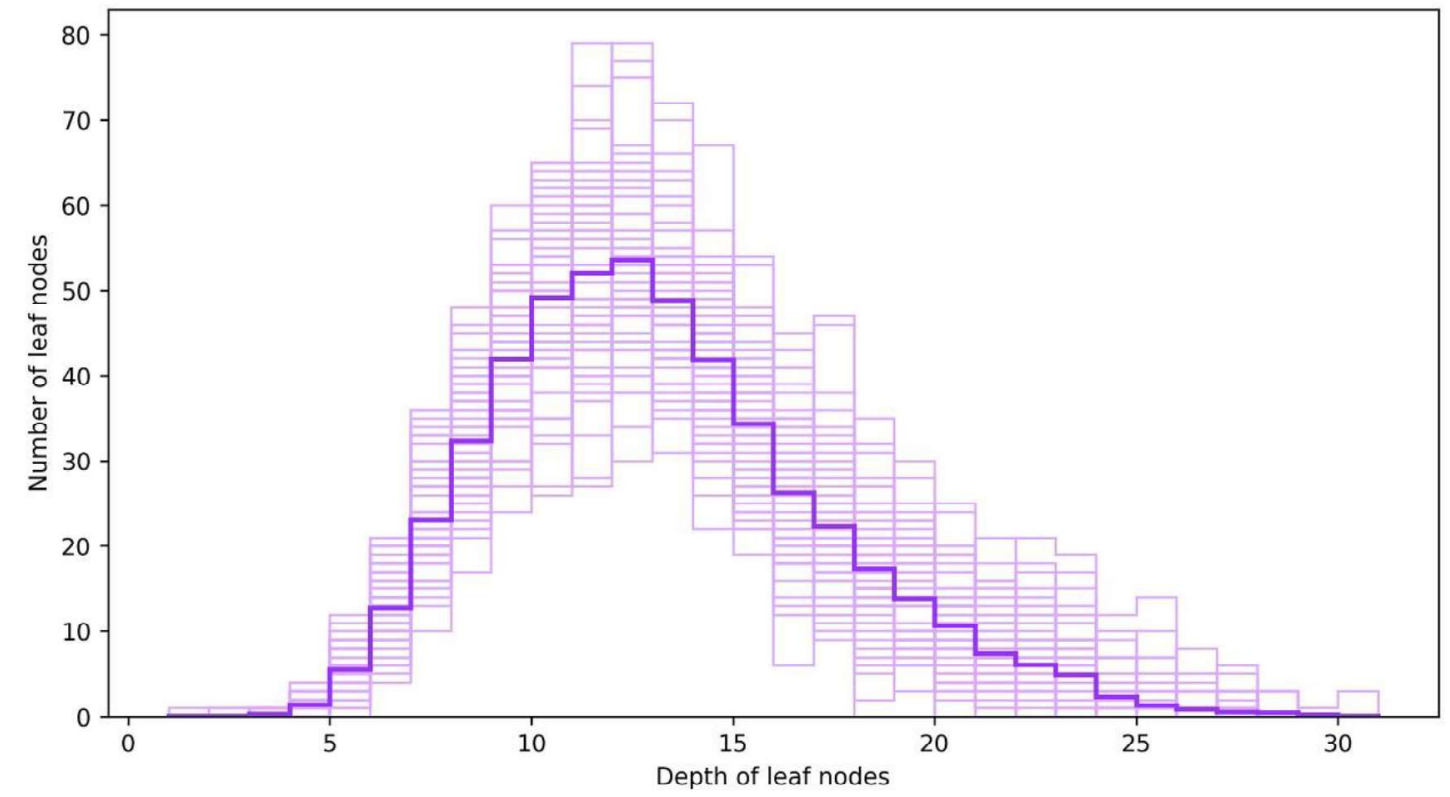

Figure 2: Visualization of depth of leaf nodes in trees

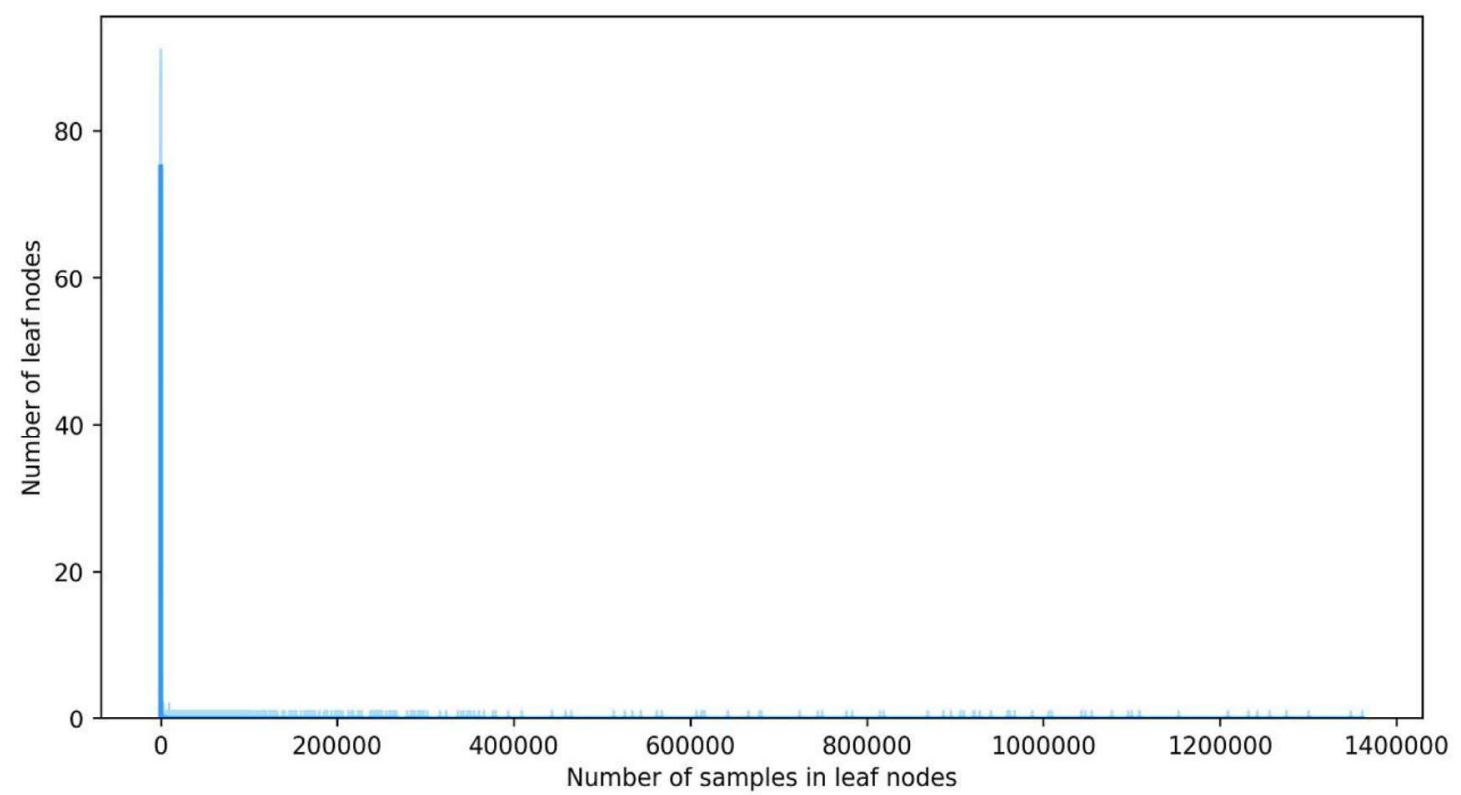

Figure 3: Visualisation of number of samples in leaf nodes in trees 
Following set of equations is used for evaluation purpose:

$T P R$ or Recall $=\frac{T P}{T P+F N}$

$F P R=\frac{F P}{F P+T N}$

Precision $=\frac{T P}{T P+F P}$

$F-$ measure $=2 . \frac{\text { Precision } * \text { Recall }}{\text { Precision }+ \text { Recall }}$

TPR in equation 9 is true positive rate which also normally known as recall. It represents the ratio between normal or any type of attack flows which are correctly detected and total number of data flows. FPR in equation 10 is false positive rate. It represents the ratio between normal or any type of attack flows which are incorrectly detected and total number of data flows. Number of normal flows is much larger than other flows so TPR and FPR can't be used for evaluation purpose. So, we need equation 11 and equation 12 for this purpose. Precision in equation 11 represents what percentage of detected attacks is correct and not a mistake. F-measure in equation 12 provides harmonic meaning of precision and recall. Ideal value for it is 1 . It'll be closer to 1 only if precision and recall are closer to 1 . F-measure value closer to 1 means rule have higher value of precision with higher value of coverage or recall.

\section{B. Achieved Results}

After applying our proposed approach on ISCX data set, 3216, 544, 8970, 967 and 1714 rules are generated for infiltrate from inside, brute force, DDoS, SSH brute force and HTTP DoS respectively. After applying evaluation method discussed in previous section, best rule for each kind of attack is obtained. TP, FP, precision, recall and f-measure for each rule generated is provided in table 5 .

Rules generated by our system are in form of "ifthen" form. "If" shows the condition and "then" shows the kind of attack. If all conditions after it happens then the attack will be detected. Some attacks use specific ports and it is prominent in our generated rules like brute force and SSH brute force attacks are performed on port 22. It can also be seen that generated rules are not only using features which were extracted from network capture but also using our generated features as well. F-measure value for HTTP DoS attack is very low and not more than $0.6544 \mathrm{~s}$. It is due to the fact that attacks designed to perform HTTP DoS for ISCX uses low bandwidth and doesn't flood the network so used features doesn't provide reliable results in this case. To achieve better and reliable results for this case, we need to consider some other features as well.

Table 5. Best rule generated for each attack

\begin{tabular}{|c|c|c|c|c|c|}
\hline Rule & TP & FP & Precision & Recall & F- \\
\hline $\begin{array}{c}\text { if flowBytesPerSec }<=83657.5 \text { and bytesPerPacket }>64.5 \text { and destinationPort }<=22 \text { and direction } \\
!=\text { L2L and destinationTCPFlags }>0 \times 2 \text { and IOByteRate }<=0.61295 \text { and totalDestinationBytes }> \\
2685.0 \text { and IOPacketRate }<=0.93095 \text { and totalSourceBytes }>1391.5 \text { then Bruteforce }\end{array}$ & 1625 & 0 & 100 & 96.44 & 0.982 \\
\hline $\begin{array}{c}\text { if totalDestinationPackets }>149.5 \text { and direction }==\mathrm{L} 2 \mathrm{~L} \text { and totalDestinationPackets }<=215.0 \text { and } \\
\text { IOPacketRate }>0.392 \text { and flowPacketsPerSec }>59.075 \text { and totalDestinationBytes }>74076 \text { and } \\
\text { totalSourcePackets }<=104.5 \text { and IOPacketRate }>0.40865 \text { then DDoS IRC }\end{array}$ & 21786 & 296 & 98.66 & 72.60 & 0.837 \\
\hline $\begin{array}{l}\text { if flowBytesPerSec }<=83657.5 \text { and direction }==\mathrm{L} 2 \mathrm{~L} \text { and destinationPort }<=143 \text { and } \\
\text { totalDestinationPackets }<=199 \text { and protocol } !=\mathrm{icmp} \text { ip and bytesPerPacket }<=66.5 \text { and } \\
\text { totalSourcePackets }>2.5 \text { and destinationTCPFlags }<=0 \times 15 \text { and totalSourcePackets }<=127.5 \text { and } \\
\text { totalSourceBytes }>195 \text { then HTTPDoS }\end{array}$ & 1493 & 11 & 99.27 & 48.80 & 0.654 \\
\hline $\begin{array}{c}\text { if direction }==\mathrm{L} 2 \mathrm{~L} \text { and totalSourcePackets }<=60.5 \text { and protocol } !=\text { udp ip and } \\
\text { totalDestinationBytes }<=514.0 \text { and bytesPerPacket }<=78.5 \text { and sourceTCPFlags }<=0 \times 10 \text { and } \\
\text { destinationPort }>110 \text { then InfilterateFrom/nside }\end{array}$ & 11097 & 0 & 100 & 67.8 & 0.808 \\
\hline $\begin{array}{l}\text { if totalDestinationPackets }<=149.5 \text { and totalSourceBytes }>65 \text { and destinationPort }==22 \text { and } \\
\text { protocol } !=\text { icmp ip and direction }==\text { R2L and IOPacketRate }<=1.6377 \text { then SSH Bruteforce }\end{array}$ & 4108 & 0 & 100 & 98.65 & 0.993 \\
\hline
\end{tabular}




\section{Comparison of Achieved Results}

This section presents the comparison of results achieved by our presented approach with other researches performed on ISCX data set. Fallahi et al. used C5.0 and ripper classifiers for this purpose [18] and their generated feature set is also different as compared to ours. Table 6 provides a brief comparison of precision value of best for our both experiments with other researches. Maximum value of precision is highlighted for each attack to give the better idea about which performs best for each attack type.

As shown in table 6, our proposed approach outperforms others in all kind of attacks except HTTP DoS attack where its performance is slightly lesser as compared to Ripper. Overall precision for rules generated by using our approach is better than others.

\section{Discussion and Analysis of Results}

Previously we presented results achieved by our approach and compared it with other researches. In this section, we will discuss what we achieved and analyze it. For brute force, infiltrate from inside and SSH brute force, we achieved $100 \%$ precision. For DDoS attack, we achieved $98.66 \%$ precision which is better than previous researches. For HTTP DoS attack, we achieved $99.26 \%$ precision which is very good but not better than previous researches as they achieved $100 \%$ precision for this specific kind of attack. Overall, our approach provides very effective rules for all kind of attacks except HTTP DoS attack and f-measure value for rules of this attack is very low. For all other kind of attacks, fmeasure value is greater than 0.80 .

\section{CONCLUSION}

This study proposes a system to generate rules for firewall using flow-based network features. Rules are generated by translating a trained machine learning classifier to firewall rules thus giving a traditional firewall characteristics of machine learning classifier which otherwise is requires very large effort. This research also suggests a method to generate firewall rules efficiently using labelled data.
A number of flow-based features are extracted from ISCX data set and on the basis of these features, six new features are generated. These features are then submitted to three different machine learning classifiers namely: logistic regression, CART, Naive Bayes, Random Forest and Ada Boost. Results from these classifiers are evaluated using 10 -fold cross validation and found that random forest provides best results. To generate rules, we used random forest classifier with 64 trees and each tree with 512 maximum nodes. After obtaining trained model, each tree in classifier is then converted to the set of rules which can then be used by traditional firewall.

The rules generated by our system have 100\% precision for most of the attack types and we achieved maximum $0.993 \mathrm{f}$-measure value. We also compared our results with other related researches and our generated rule out perform others in terms of precision and f-measure for four kind of attack while for one type of attack, our rule's performance is slightly lesser as compared to others. Our proposed work has significant value in two ways:

1. Given a very large labelled data, this system can convert it to firewall rules with minimum time and effort. It isn't restricted to flow-based features only but can generate rules using even content-based features.

2. Traditional firewall has very large communities working for updating rules for new attacks but they can't keep pace with new attacks and these firewalls also lack ML classification characteristics. On the other hand, anomaly detection solutions are using ML for this purpose but those solutions are not good enough yet to be deployed independently. Our approach provides a hybrid solution. By translating trained model for anomaly detection to firewall rules, we are actually integrating characteristics of anomaly detector in traditional firewall in some sense.

Currently we are using ISCX data set for classification purposes which generated very efficient rules but in future many more data sets can be used to test how efficient and effective this approach can be. Moreover, we are only using flow- 
based features. Rules generated by using these features are very efficient and requires very small computation power for detection purposes but still these features are not enough for detection of many kind of attacks. In future, content-based features can also be used for rule generation which will make the rules much more powerful and can cover many more kind of attack which are impossible to detect by using just flow-based parameters.

\section{References}

[1] Tamas Abraham. Iddm: Intrusion detection using data mining techniques. 2001.

[2] James P Anderson et al. Computer security threat monitoring and surveillance. Technical report, Technical report, James P. Anderson Company, Fort Washington, Pennsylvania, 1980.

[3] AV-TEST - The Independent IT-Security Institute. Malware statistics \& trends report, Last accessed on June 18, 2017.

[4] Monowar H Bhuyan, Dhruba Kumar Bhattacharyya, and Jugal K Kalita. Network anomaly detection: methods, systems and tools. Ieee communications surveys \& tutorials, 16(1):303-336, 2014.

[5] Carson Brown, Alex Cowperthwaite, Abdulrahman Hijazi, and Anil Somayaji. Analysis of the 1999 darpa/lincoln laboratory ids evaluation data with netadhict. In Computational Intelligence for Security and Defense Applications, 2009. CISDA 2009. IEEE Symposium on, pages 1-7. IEEE, 2009.

[6] C. Catania and C. Garca Garino. Towards reducing human effort in network intrusion detection. In 2013 IEEE 7th International Conference on Intelligent Data Acquisition and Advanced Computing Systems (IDAACS), volume 02, pages 655-660, Sept 2013.

[7] F. Lydia Catherine, R. Pathak, and V. Vaidehi. Efficient host-based intrusion detection system using partial decision tree and correlation feature selection algorithm. In 2014 International Conference on Recent Trends in Information Technology, pages 1-6, April 2014.

[8] Hee-su Chae, Byung-oh Jo, Sang-Hyun Choi, and Twae-kyung Park. Feature selection for intrusion detection using nsl-kdd. Recent Advances in Computer Science, pages 184-187, 2013.

[9] Hui-Hao Chou and Sheng-De Wang. An adaptive network intrusion detection approach for the cloud environment. In Security Technology (ICCST), 2015 International Carnahan Conference on, pages 1-6. IEEE, Sept 2015.

[10] IOS Cisco. Netflow configuration guide release 12.4. Cisco Documentation, Cisco Systems, San Jose, 2007.

[11] B Claise. Rfc 3954: Cisco systems netflow services export version 9 (2004). Last accessed on June 18, 2017.

[12] Benoit Claise, Brian Trammell, and Paul Aitken. Specification of the ip flow information export (ipfix) protocol for the exchange of flow information. Technical report, 2013.

[13] Dorothy E Denning. An intrusion-detection model. IEEE Transactions on software engineering, (2):222-232, 1987.

[14] L Dhanabal and SP Shantharajah. A study on nslkdd dataset for intrusion detection system based on classification algorithms. International Journal of Advanced Research in Computer and Communication Engineering, 4(6):446-452, 2015.

[15] Roberto Di Pietro and Luigi V Mancini. Intrusion detection systems, volume 38. Springer Science \& Business Media, 2008.

[16] Sumeet Dua and Xian Du. Data mining and machine learning in cybersecurity. CRC press, 2016.

[17] Ilhame El Farissi, Sara Chadli, Mohamed Emharraf, and Mohammed Saber. The analysis of kdd-parameters to develop an intrusion detection system based on neural network. In Advances in Ubiquitous Networking 2, pages 491-503. Springer, 2017.

[18] N. Fallahi, A. Sami, and M. Tajbakhsh. Automated flow-based rule generation for network intrusion detection systems. In 2016 24th Iranian Conference on Electrical Engineering (ICEE), pages 1948-1953, May 2016.

[19] Mike Fisk and George Varghese. Fast contentbased packet handling for intrusion detection. Technical report, DTIC Document, 2001. 
[20] Alexandros G Fragkiadakis, Vasilios A Siris, Nikolaos E Petroulakis, and Apostolos P Traganitis. Anomalybased intrusion detection of jamming attacks, local versus collaborative detection. Wireless Communications and Mobile Computing, 15(2):276-294, 2015.

[21] D. P. Gaikwad and R. C. Thool. Intrusion detection system using bagging ensemble method of machine learning. In 2015 International Conference on Computing Communication Control and Automation, pages 291-295, Feb 2015.

[22] Ming Gao, Kenong Zhang, and Jiahua Lu. Efficient packet matching for gigabit network intrusion detection using tcams. In Advanced Information Networking and Applications, 2006. AINA 2006. 20th International Conference on, volume 1, pages 6-pp. IEEE, 2006.

[23] E. Godefroy, E. Totel, M. Hurfin, and F. Majorczyk. Automatic generation of correlation rules to detect complex attack scenarios. In 2014 10th International Conference on Information Assurance and Security, pages 23-28, Nov 2014.

[24] Dina Hadziosmanovi` c, Lorenzo Simionato, Damiano Bolzoni, Emmanuele Zambon, and Sandro Etalle. N-' gram against the machine: On the feasibility of the n-gram network analysis for binary protocols. In International Workshop on Recent Advances in Intrusion Detection, pages 354-373. Springer, 2012.

[25] Jason Hong. The state of phishing attacks. Communications of the ACM, 55(1):74-81, 2012.

[26] Neminath Hubballi and Vinoth Suryanarayanan. False alarm minimization techniques in signaturebased intrusion detection systems: A survey. Computer Communications, 49:1-17, 2014.

[27] MA Jabbar, Rajanikanth Aluvalu, and S Reddy. Cluster based ensemble classification for intrusion detection system. In Proceedings of the 9th International Conference on Machine Learning and Computing, pages 253-257. ACM, 2017.

[28] Kamarularifin Abd Jalil, Muhammad Hilmi Kamarudin, and Mohamad Noorman Masrek. Comparison of machine learning algorithms performance in detecting network intrusion. In Networking and Information Technology (ICNIT), 2010 International Conference on, pages 221-226. IEEE, June 2010.
[29] V. Jyothi, X. Wang, S. K. Addepalli, and R. Karri. Brain: Behavior based adaptive intrusion detection in networks: Using hardware performance counters to detect ddos attacks. In 2016 29th International Conference on VLSI Design and 2016 15th International Conference on Embedded Systems (VLSID), pages 587-588, Jan 2016.

[30] Minsoo Kim, Jae-Hyun Seo, Il-Ahn Cheong, and Bong-Nam Noh. Auto-generation of detection rules with tree induction algorithm. Fuzzy Systems and Knowledge Discovery, pages 484 484, 2005.

[31] Haiguang Lai, Shengwen Cai, Hao Huang, Junyuan $\mathrm{Xie}$, and Hui Li. A parallel intrusion detection system for high-speed networks. In International Conference on Applied Cryptography and Network Security, pages 439-451. Springer, 2004.

[32] Van Lam Le, Ian Welch, Xiaoying Gao, and Peter Komisarczuk. Anatomy of drive-by download attack. In Proceedings of the Eleventh Australasian Information Security Conference-Volume 138, pages 49-58. Australian Computer Society, Inc., 2013.

[33] Hung-Jen Liao, Chun-Hung Richard Lin, YingChih Lin, and Kuang-Yuan Tung. Intrusion detection system: A comprehensive review. Journal of Network and Computer Applications, 36(1):16-24, 2013.

[34] Wei-Chao Lin, Shih-Wen Ke, and Chih-Fong Tsai. Cann: An intrusion detection system based on combining cluster centers and nearest neighbors. Knowledge-based systems, 78:13-21, 2015.

[35] Lincoln Laboratory MIT. Darpa intrusion detection evaluation, Last accessed on May 27, 2017.

[36] Wei-Yin Loh. Classification and regression trees. Wiley Interdisciplinary Reviews: Data Mining and Knowledge Discovery, 1(1):14-23, 2011.

[37] Matthew V Mahoney and Philip K Chan. Learning nonstationary models of normal network traffic for detecting novel attacks. In Proceedings of the eighth ACM SIGKDD international conference on Knowledge discovery and data mining, pages 376385. ACM, 2002.

[38] John McHugh. Testing intrusion detection systems: A critique of the 1998 and 1999 darpa intrusion detection system evaluations as performed by lincoln laboratory. ACM Trans. Inf. Syst. Secur., 3(4):262-294, November 2000. 
[39] B. Mukherjee, L. T. Heberlein, and K. N. Levitt. Network intrusion detection. IEEE Network, 8(3):26-41, May 1994.

[40] Open Information Security Foundation. suricata, July 2010 .

[41] Swati Paliwal and Ravindra Gupta. Denial-ofservice, probing \& remote to user (r21) attack detection using genetic algorithm. International Journal of Computer Applications, 60(19):57-62, 2012.

[42] Vern Paxson. Bro: a system for detecting network intruders in real-time. Computer networks, 31(23):2435-2463, 1999.

[43] F. Pedregosa, G. Varoquaux, A. Gramfort, V. Michel, B. Thirion, O. Grisel, M. Blondel, P. Prettenhofer, R. Weiss, V. Dubourg, J. Vanderplas, A. Passos, D. Cournapeau, M. Brucher, M. Perrot, and E. Duchesnay. Scikit-learn: Machine learning in Python. Journal of Machine Learning Research, 12:2825-2830, 2011.

[44] YP Raiwani and Shailesh Singh Panwar. Data reduction and neural networking algorithms to improve intrusion detection system with nsl-kdd dataset. International Journal of Emerging Trends \& Technology in Computer Science, 4(1):219-225, 2015.

[45] R. Rajpal, S. Kaur, and R. Kaur. Improving detection rate using misuse detection and machine learning. In 2016 SAI Computing Conference (SAI), pages 1131-1135, July 2016.

[46] Martin Roesch et al. Snort: Lightweight intrusion detection for networks. In Lisa, volume 99, pages 229-238, 1999.

[47] Ganesh Sadasivan. Architecture for ip flow information export. Last accessed on June 18, 2017.

[48] Karen Scarfone and Peter Mell. Guide to intrusion detection and prevention systems (idps). NIST special publication, 800(2007):94, 2007.

[49] scikit-learn developers. Decision trees, Last accessed on June 4, 2017.

[50] Kyu-Seok Shim, Sung-Ho Yoon, Su-Kang Lee, Sung-Min Kim, Woo-Suk Jung, and Myung-Sup Kim. Automatic generation of snort content rule for network traffic analysis. The Journal of Korean
Institute of Communications and Information Sciences, 40(4):666 - 677, 2015.

[51] Ali Shiravi, Hadi Shiravi, Mahbod Tavallaee, and Ali A Ghorbani. Toward developing a systematic approach to generate benchmark datasets for intrusion detection. computers \& security, 31(3):357-374, 2012.

[52] Akhilesh Kumar Shrivas and Amit Kumar Dewangan. An ensemble model for classification of attacks with feature selection based on kdd99 and nsl-kdd data set. International Journal of Computer Applications, 99(15), 2014.

[53] Robin Sommer and Vern Paxson. Outside the closed world: On using machine learning for network intrusion detection. In Security and Privacy (SP), 2010 IEEE Symposium on, pages 305-316. IEEE, 2010.

[54] A. Sperotto, G. Schaffrath, R. Sadre, C. Morariu, A. Pras, and B. Stiller. An overview of ip flowbased intrusion detection. IEEE Communications Surveys Tutorials, 12(3):343-356, Third 2010.

[55] Mahbod Tavallaee, Ebrahim Bagheri, Wei Lu, and Ali A Ghorbani. A detailed analysis of the kdd cup 99 data set. In Computational Intelligence for Security and Defense Applications, 2009. CISDA 2009. IEEE Symposium on, pages 1-6. IEEE, 2009.

[56] Mahbod Tavallaee, Natalia Stakhanova, and Ali Akbar Ghorbani. Toward credible evaluation of anomalybased intrusion-detection methods. IEEE Transactions on Systems, Man, and Cybernetics, Part C (Applications and Reviews), 40(5):516-524, 2010.

[57] T. Werner, C. Fuchs, E. Gerhards-Padilla, and P. Martini. Nebula - generating syntactical network intrusion signatures. In 2009 4th International Conference on Malicious and Unwanted Software (MALWARE), pages 31-38, Oct 2009.

[58] Handong Wu, Stephen Schwab, and Robert Lom Peckham. Signature based network intrusion detection system and method, September 92008. US Patent 7,424,744.

[59] T. Xing, D. Huang, L. Xu, C. J. Chung, and P. Khatkar. Snortflow: A openflow-based intrusion prevention system in cloud environment. In 2013 Second GENI Research and Educational Experiment Workshop, pages 89-92, March 2013. 
[60] Ali, Mohammed \& Saudi, Madihah \& Bhuiyan, Touhid \& Bakar, Azreena. (2018). Comparative Study of Traditional and Next Generation IPS. International Journal of Engineering and Technology (UAE). $7 . \quad 55-58$. 10.14419/ijet.v7i4.15.21371. October 2018.

[61] Asharf, J.; Moustafa, N.; Khurshid, H.; Debie, E.; Haider, W.; Wahab, A. A Review of Intrusion Detection Systems Using Machine and Deep Learning in Internet of Things: Challenges, Solutions and Future Directions. Electronics 2020, 9, 1177.

[62] Ahmad, Z, Shahid Khan, A, Wai Shiang, C, Abdullah, J, Ahmad, F. Network intrusion detection system: A systematic study of machine learning and deep learning approaches. Trans Emerging Tel Tech. 2020; e4150. https://doi.org/10.1002/ett.4150

[63] Riyaz, B., Ganapathy, S. A deep learning approach for effective intrusion detection in wireless networks using CNN. Soft Comput 24, 1726517278 (2020). https://doi.org/10.1007/s00500-02005017-0

[64] S. T. Zhang, X. B. Lin, L. Wu, Y. Q. Song, N. D. Liao, Z. H. Liang, "Network Traffic Anomaly Detection Based on ML-ESN for Power Metering System", Mathematical Problems in Engineering, vol. 2020, Article ID 7219659, 21 pages, 2020. https://doi.org/10.1155/2020/7219659 